\title{
Temi Musicali e Temi Ebraici nel Pensiero di Wladimir Jankélévitch
}

\author{
Enrico Fubini
}

Wladimir Jankélévitch, filosofo e musicologo, ha dedicato una parte relativamente esigua delle sue riflessioni ad un tema in apparenza secondario ed eterogeneo rispetto ai suoi interessi dominante, cioè alla situazione esistenziale dell'ebreo diasporico nel mondo odierno, delineando così una fenomenologia della coscienza ebraica. Nella immensa produzione filosofica di Jankélévitch tale riflessione occupa una posizione senza dubbio marginale ma tuttavia, dal momento che la sua personalità non ha i caratteri dell' eclettismo, sorge spontanea la domanda se le sue riflessioni sull'ebraismo s'inseriscano nel contesto più generale del suo pensiero o se rimangano un episodio isolato, legato a sue vicende biografiche personali. L'attenzione alla tematica ebraica, divenuta in lui viva soprattutto dopo la guerra, concretatasi in un appassionato impegno di saggista non solo sui problemi di attualità più bruciante come le Stato d'Israele o il drammatico confronto con i crimini nazisti e il problema del perdono, ma anche sui più astratti e filofofici temi della natura della coscienza ebraica, del diritto alla diversità ecc., sembra a tutta prima del tutto scissi dai suoi ben noti interessi musicali. Va inoltre ricordato che gli interessi musicali di Jankélévitch sono estremamente settoriali e si concentrano su pochi autori (pra-

* Agradecimentos ao Instituto Italiano de Cultura co-responsável pela visita de Enrico Fubini ao Departamento de Música da ECA-USP em 1995. 
ticamente su Fauré, Debussy e Ravel) ed hanno una natura quindi eminentemente ideologica. In altre parole, ci si chiede, tra la saggistica musicale di Jankélévitch e le sue riflessioni sull'ebraismo è individuabile una qualche intrinseca relazione, un filo rosso che colleghi i due campi, in apparenza così lontani ed estranei l'uno all'altro, cioè una continuità di pensiero?

Per rispondere a questo quesito è forse necessario ripercorrere brevemente i punti più salienti del pensiero musicale di Jankélévitch per ritrovare le coordinate estetiche e più in generale filosofiche che lo hanno guidato nella sua speculazione. Si è detto che i musicisti prediletti da Jankélévitch sono scelti da una rosa assai ristretta di autori francesi a cavallo tra romanticismo, impressionismo e simbolismo. Chiaramente una scelta così circostanziata e precisa è dettata non solo da una preferenza sul piano estetico ma da una ben precisa scelta ideologica. Per brevità si può senz'altro affermare che la musica di Debussy rappresenta il centro della speculazione estetica e filosofica di Jankélévitch, e quindi scavando all'interno di tale scelta si ritrovano le ragioni più profonde del suo pensiero.

Perché dunque proprio Debussy? La musica di Debussy, secondo Jankélévitch, rappresenta la più straordinaria incarnazione artistica di una concezione non architettonica e spaziale del tempo ma piuttosto organica e vitalistica, vicina in qualche modo alla concezione bergsoniana del tempo. Forse si può ancora aggiungere che tale modo di sentire e vivere il tempo come durata non può che incarnarsi in una forma musicale. "La musica di Debussy elimina in tal modo il principio di una sostanza centrale gerarchicamente prima e compiutamente rappresentabile (como invece avveniva nell'arte tradizionale), per sostituirvi una nova entrevision della realtà, quale 'innumerabile' e inafferrabile apparenza di una polvere di molecole instantanee, di schegge luminose che quiora splendono. Alla fuidità faureana, bergsoniana del tempo, nonché alla scansione razionalistica di esso come processo snodato in sequenze dialettiche (slanci e risoluzioni) è opposta una 'disgregazione' fatta di abbaglianti folgorazioni, di 'immediate epifanie risplendenti"' Così, uno dei più acuti critici italiani di Jankélévitch, evidenzia ciò che 
per il filosofo francese è il nucleo centrale dell'arte di Debussy e soprattutto la sua portata ideologica nell'ambito della musica occidentale. Infatti Debussy incarna una delle più profonde ribellione al logos, come ragione dialettica, consequenziale e onnicomprensiva, di cui la forma sonata, nella musica, rappresentava la più compiuta e perfetta incarnazione; al tempo stesso addita una nuova via, alternativa, alla musica stessa in cui tutti i valori fortemente affermativi della musica precedente vengono in qualche modo rovesciati in nome di una nuova poetica della marginalità e del silenzio. Se nella tradizione romantico-ottocentesca erano considerati valori positivi la conclusività e l'affermatività realizzati con la forza del meccanismo cadenzale, la complessità costruttiva realizzata innanzitutto con la struttura sonatistica, la dialettica delle parti che approda ad una sintesi onnicomprensiva e rassicurante capace di superare tutte le apparenti contraddizioni, il "progresso" che si snoda attraverso sicuri percorsi nello spazio e che si sviluppa secondo una ferrea logica musicale, tutti questi valori nella musica di Debussy vengono radicalmente capovolti. La musica, per sua natura, in quanto "linguaggio defluente e incoerente, equivoco e discontinuo, spezza la coerenza verbosa del Logos rappresentativo, sgretolandone l'impianto ontologico totalitario e alludendo ad uno completamente altro, destituito di qualsiasi fondamento sostanziale. Nella musica c'è allusione ad un regime ontologico - un essere delle cose - né rassicurato né rassicurante, ma del tutto incerto, dubitativo, incessantemente penetrato di non essere, interrotto dalla morte, trascinato tra essere e non essere: sempre sulla soglia quasi del nulla: presque rien. Ma un (non) essere allora che è piuttosto un divenire. Là dove divenire va inteso non come un cammino diritto e unilineare, sorretto da una precisa strategia, bensi come un puro essere in moto...."2, Cosi commenta ancora Enrica Lisciani-Petrini il pensiero di Jankélévitch. Ora c'è da chiedersi perché, la musica, quella di Debussy per intenderci, che rappresenta questa alternativa radicale alle "ebbrezze razionalistiche" della civiltà occidentale, non si è sempre posta in questi termini, ma invece per lungo tempo, per lo meno in Occidente, ha accompagnato e favorito l'ideale 
rassicurante e progressivo in cui siamo cresciuti. Il linguaggio musicale, per sua natura inesprimibile e ineffabile si sarebbe orientato verso questa inespressività originaria, se non fosse stato artificialmente piegato ad una concezione del mondo espressiva e rassicurante. Nel mondo odierno, in cui tutte le razionali e rassicuranti certezze di ieri sono entrate in crisi, la musica, ma non solo la musica, è diventata in qualche modo il simbolo di un altro pensiero, di un diverso stato di coscienza, di un diverso modo di vivere il tempo.

Il discorso sulla musica rimanda pertanto a problemi di altra natura e forse si può cominciare ora ad intravvedere il suo segreto legame con l'ebraismo. Scorrendo, anche distrattamente, gli scritti ebraici di Jankélévitch si rimane a tutta prima colpiti di notare che in essi ricorrono termini e concetti che già comparivano nei suoi scritti musicali ${ }^{3}$. Anzitutto anche per quanto riguarda l'ebraismo è centrale la riflessione sul tempo e sulla modalità ebraica di vivere il tempo, soprattuto in relazione all'idea messianica. Il rifiuto dello storicismo e della dialettica consolatoria e conciliante costituiscono un altro asse portante della sua riflessione; ma stupisce altresi di trovare la stessa terminologia quale "indefinito", "inesprimibile", "non so che", "elusività", "differenza" ecc. di cui già si serviva per descrivere lo status ontologico della musica. Ciò forse potrebbe semplicemente spiegarsi con il fatto che l'autore è affezionato ad una certa terminologia e che quindi questa ricorre in tutti gli scritti, di qualsiasi argomento trattino. Ma in un filosofo così raffinato ed acuto qual'è Jankélévitch ci sembra una spiegazione troppo riduttiva. L'uso di una medesima terminologia meglio si spiega con l'ipotesi che ci si trova di fronte a problemi che ad un'attenta analisi revelano una profonda affinità.

L'esistenza ebraica è spiegata da Jankélévitch in termini di sottili ambivalenza per non dire di sottile e inafferrabile ambiguità. Ci sono elementi di insanabile contraddizione nella stessa struttura concettuale della vita ebraica: l'esilio, in questa prospettiva, è visto non come un evento storico, superabile in virtù di altri possibili eventi storici, ma inscindibilmente conesso alla stessa coscienza 
ebraica. Così si esprime acutamente Jankélévitch in un suo saggio del 1957: "Il movimento rappresenta la maniera di esistere di una coscienza che porta in se stessa degli elementi contraddittori, il modo di essere di un uomo che è straniero e indigeno, che vuole a un tempo assomigliare e 'dissomigliare'. Il peregrinare, che si oppone alla tendenza all' insediamento, fu sempre considerato come uno dei tratti fondamentali di Israele"4. Il movimento a cui si allude non è un movimento nello spazio, ma è da riportarsi al tempo, un movimento perciò che è legato all'idea del divenire. Ma, afferma ancora Jankélévitch, "lo spirito di movimento fa d'Israele il portatore privilegiato della contraddiozione umana.... Un non so che di definito e di indefinibile si esprime in questo problema irritante, senza sosta risolto, sensa sosta rinascente e, per farla breve, essenzialmente equivoco"s. La situazione dell'ebreo è dunque irrimediabilmente ambigua e trae la sua linfa vitale dall' irresolubilità delle contradizione all'interno delle quali egli vive. "Di qui - continua Jankélévitch viene forse lo spirito di mobilità di cui Israele è il portatore. Il movimento è la sola soluzione alla tensione interiore, così come il divenire, che è la nostra vocazione, risolve la contraddizione dell'essere e del non essere, l'uomo diviene questi opposti che egli non può essere simultaneamente". Non vi è conciliazione dunque, le contraddizioni rimangono aperte, le lacerazioni non sono sanabili ma sono feconde, sono il sale della terra.

Scorrendo con attenzione le pagine del filosofo sulla coscienza ebraica ci si accorge che in esse viene privilegiato un aspetto particolare dell'ebraismo: la condizione diasporica, condizione invalicabile, fonte di dolore e d'infelicità ma al tempo stesso di feconda e produttiva inquietudine. La conclusione di Jankélévitch è centrale per il nostro discorso: "La lacerazione ebraica, in questo, è una forma privilegiata della lacerazione umana in generale"7, ed aggiunge: "La peculiarità dell' ebreo non è mai stata quella di cercare la soluzione nella sintesi conciliatrice: lasciamo questo compito a Hegel e ai suoi amici. Crediamo piuttosto alla fecondità di un'oscillazione vibratoria infinita fra questi due poli: la disseminazione con la sua inquietudine, la Diaspora che è principio 
d'aporia; dall'altra parte, lo Stato temporale, certamente banale come tutti gli altri Stati, ma che rappresenta la possibilità intramondana di Israele, che dico?, la sua certezza quaggiù, l'affermazione stessa della sua pienezza vitale"8. Pertanto il dualismo e l'oscillazione, così presente ieri ma anche oggi, nell' animo ebraico tra la diaspora e un Israele, ieri lontano, immaginato, sconosciuto, desiderato, invocato, oggi reale e presente nella sua concretezza, "non è un vicolo cieco disperante, ma è una polarità vivificante che eletrizza la coscienza ebraica"9. A chi rimprovera agli ebrei di non accettare senza riserve né l'assimilazione totale né la nazionalità israeliana, Jankélévitch risponde: “.... non siamo noi a non sapere ciò che vogliamo, è la verità che è lacerata e incoerente, sono le verità che sono sporadiche e incompatibili e non possono essere onorate tutte insieme"10. Così conclude Jankélévitch in un altro saggio dal significativo titolo, L'ebraismo, problema interiore, affermando che la situazione esistenziale dell'ebreo "è inquietante anche quando non è tragica, è una sollecitazione a cercare sempre altrove, sempre oltre.... C'è una perplessità infinita, che non comporta né fine né soluzione. Io non conosco il modo di fare in me stesso la sintesi delle contraddizioni, e la conciliazione hegeliana ha per noi poca attrattiva. La nostra perplessità durerà fino alla fine dei tempi, i quali non hanno fine""11.

Non tutti possono concordare su questa concezione dell'ebraismo che privilegia un aspetto senza dubbio centrale, cioè il carattere diasporico, visto come la principale molla propulsiva dell'ebraismo stesso. Un ipotetico ritorno di tutti gli ebrei in Israele sarebbe considerato da Jankélévitch come la fine dell'ebraismo o meglio la fine di quella situazione esistenziale dell'ebreo a lui così cara cioè di quella produttiva e feconda tensione di cui si è sempre alimentato l'animo ebraico, l'avvento quindi di una normalità nella storia che cancellerebbe la diversità ebraica. Ma non è il luogo qui per mettere in questione questa visione dell'ebraismo: ci basta ricordare che è la sua visione dell' ebraismo e che ve non sono altre in cui la diaspora stessa viene vista come un accidente storico, deprecabile, gravido di pericoli che può, oggi in particolare, mettere 
in forse l'esistenza stessa dell'ebraismo.

Ancora un cenno, per meglio penetrare nel suo ebraismo, ad un problema strettamente connesso a quelli a cui già si è fatto cenno: il messianismo ebraico. Nota Jankélévitch che si possono dare due diversi tipi di messianismo. Il primo implica l'idea della fine dei tempi: l'avvento del Messia segnerebbe un termine a cui prima o poi si giunge, per lontano che sia; il tempo verrebbe a configurarsi come "una grandezza scalare", come un "mobile che avanza regolarmente e sempre nello stesso senso" e "il diveniente si avvicina sempre più alla consumazione del tempo"12. La storia significherebbe dunque srotolare "il rotolo dei possibili" e si fermerebbe quando il rotolo è alla fine; questo vale anche per la vita umana: "noi siamo degli esseri limitati che abbiamo solo pochissime idee: ci fermiamo quando siamo in fondo, come si ferma un orologio che esaurisce la carica"13. Il secondo modo d'intendere il messianismo è proprio dell'ebraismo e anzitutto implica l'abbandono dell'idea di un Messia personale che giunge in un tempo determinato: "Fin dal libro di Isaia gli ebrei hanno voltato le spalle all'idea di un Messia personale, per fare del messianismo il campo di una speranza pneumatica, indeterminata, di natura così morale e religiosa da implicare una trasfigurazione morale degli uomini. A questa trasfigurazione ci si guarda bene dall'assegnare una data. La depersonalizzazione del Messia, rimasto personale solo nelle credenze popolari, è un fenomeno essenziale nella storia filosofica dell'ebraismo" ${ }^{14}$. Il tempo assume dunque un carattere d'infinitezza e il vero problema non è la fine dei tempi, ma piuttosto "i fini del tempo"; "non la fine della storia, perché essa non avrà mai fine, perché non sarà mai alla fine del suo rotolo; il nostro vero problema sono i fini della storia; i fini smentiscono la fine; i fine che sono ideali, normativi, con i quali viviamo, che sono capaci di produrre in noi i grandi rinnovamenti e che non ci lasciano la possibilità di dire 'per sempre'... Per il fatto stesso che noi ci rivolgiamo a un futuro infinitamente lontano, che questo futuro non arriverà mai sul calendario, ma sopraggiungerà come un profondo mistero, come il mistero della morte sbocca in un altro ordine, esso è sempre presente: dire che è sempre futuro o 
sempre presente è la stessa cosa... Questo domani è il mio oggi, brilla in questa lampada accesa della speranza che è nel cuore di ciascuno di noi e trasfigura il nostro 'ogni giorno', la nostra quotidianità, l'orienta perché la speranza fiorisca continuamente in noi" 15 . Così Jankélévitch conclude il denso saggio del 1961, La speranza e la fine dei tempi, saggio sul messianismo ebraico ma più ancora saggio sul senso del tempo nell'ebraismo.

Ed infine ancora un accenno ad un altro importante saggio Assomigliare, dissomigliare, del 1964. Anche qui Jankélévitch mette ancora una volta il dito su quella contraddizione irrisolta e irresolubile che sta alla radice dell'esistenza ebraica ma che al tempo stesso diventa una cifra universale dell'esistenza umana. L'ebreo è costantemente preso tra due tentazioni diverse ed opposte, speculari una all'altra: essere come gli altri, cioè assomigliare, essere come tutti, o isolarsi nella propria specificità, diversità e particolarità. Si potrebbe universalizzare questa duplice tentazione affermando che una rispecchia il bisogno di vita sociale, l'altra il bisogno di solitudine che si manifesta anche come "la protesta dell'uomo che non vuole scomparire in questo universale grigiore": l'uomo ebreo è alla ricerca di questa forte particolarità, ma allo stesso tempo "sente la nostalgia dell' apertura, di tutto ciò a cui egli si chiude, di tutto ciò a cui rinuncia, e ha la sensazione di rinchiudersi e di impoverirsi" ". Un dilemma non molto diverso si rivela nel "volere allo stesso tempo essere trattato assolutamente come gli altri, senza alcuna discriminazione" ma conservando al tempo stesso la propria originalità. "E tuttavia è questa rassomiglianza differente o questa differenza rassomigliante che spiega il lato problematico dell' ebreo, il suo senso enigmatico", e così "sfugge col movimento, sfugge con l'umorismo e sfugge essendo un altro da se stesso, all'infinito"17. Ed ancora per concludere: "Chi non accetta di esse come gli altri né un altro dagli altri, chi non accetta di essere uno fra loro né il solo della sua specie, accetta di essere un altro da sé sviluppandosi all'infinito, sfuggendo a se stesso"18. Quale atteggiamento di fronte a questa duplice tentazione, al desiderio di assomigliare e al contro-desiderio di dissomigliare? "Non è serio - afferma ancora 
Jankélévitch - essere tentati dal contro-desiderio del proprio desiderio. Non è una cosa seria né una verità. $E^{\prime}$ qualcosa, al contrario, che richiede di essere trattato come uno scherzo, con umorismo. E' serio, al contrario, riconoscere al limite il lato un po' ironico della nostra condizione, il lato contraddittorio e ironico della nostra condizione di uomini. C'è una perfetta serietà che non ne ha l'aria, quella dell' ebreo che va avanti, che raggiunge in questo modo l'innocenza, lontano da ogni tentazione impura... L'uomo che ha capito, che si è convertito a questa innocenza - e forse la coscienza ebraica vi è particolarmente preparata ed ha in questo senso un valore esemplare --, l'uomo che ha capito il valore dell'innocenza, e il lato illusorio e puerile di queste tentazioni che egli stesso ha fabbricato, riconoscerà che tutto ciò non valeva la pena, che non vale veramente la pena lasciarsi tentare dal serpente per così poche cose. Capirà che il frutto proibito, una volta che non è più proibito, ha un gusto molto amaro, lo getterà lontano da sé e arrossirà un giorno di averlo tanto desiderato"19.

Questi passi sopra citati permettono di cogliere in modo sufficientemente chiaro il modo d'intendere l'ebraismo da parte di Jankélévitch e di ritornare quindi al discorso iniziale sulla musica. Abbiamo parlato di terminologia affine: appare ora chiaro che vi è una ragione profonda del fatto che tale terminologia, o meglio, tali strumenti concettuali, si possono perfettamente a buon diritto applicare a questi due campi, la musica e l'ebraismo, in quanto essi rivelano, ad un attenta analisi, una profonda affinità strutturale. Per la musica "l'equivoco è il regime normale" e perciò essa "non è tenuta a scegliere fra sentimenti contraddittori" ma anzi "compone con essi - ad onta di ogni alternativa - un solo stato d'animo complessivo, uno stato d'animo ambivalente e sempre indefinibile"20. La contraddizione non risolta e irresolubile è dunque l'anima della musica, della musica autentica, come può essere per l'appunto quella di Debussy. Ma tale musica, tale esperienza esistenziale privilegiata diventa, proprio come l'ebraismo, una cifra dell'esistenza umana, una spia per cogliere i lati più autentici e profondi della stessa condizione umana. Si può così enumerare tutta una serie di parentele 
tra musica ed ebraismo, rimanendo ovviamente all'interno dell'esperienza di pensiero di Jankélévitch. Debussy ha sottolineato con la sua musica la condizione dell'uomo che rinuncia alla saldezza dell'esistenza, al suo significato univoco e affermativo: la realtà non è più sentita come la casa dell'uomo, la patria salda in cui ci si riconosce senza residui. Debussy con la sua musica ha sancito la condizione della Heimatlosigkeit, della perdita della terra e della patria, propria dell'uomo. Come non avvertire la non casuale coincidenza con l'ebreo che vive ormai un esistenza costituzionalmente priva di patria e che al tempo stesso anela ad una patria, preso da un eterna e inestinguibile nostalgia di patria, che neppure la nascita dello Stato d'Israele ha potuto estinguere? Cosi l'illusoria concretezza univoca del reale è stata spazzata via dalla musica di Debussy e dalla sua ineffabile inespressività ed ambiguità. "La musica - afferma Jankélévitch - discorso vago e defluente, si situa dunque al di là delle categorie separate del comico e del tragico, nella profondità stessa della vita vissuta"21. La definitezza non si confa' alla musica che per sua natura tende ad 'esprimere l'inesprimibile all'infinito' e il suo ambito "non è l'indicibile ma l'ineffabile. L'indicibile è la notte nera della morte, perché essa è tenebra impenetrabile e desolante non essere... Mentre l'ineffabile, del tutto all'opposto, è inesprimibile perché su di esso c'è infinitamente, interminabilmente da dire..."22. Anche a questo proposito si può constatare la perfetta specularità tra l'esperienza musicale e l'esperienza ebraica. Anche l'ebraismo ha una sua ineffabilità perché definirlo è "come definire qualcosa la cui essenza è quella di essere indefinibile"23. L'ebreo infatti vuole essere se stesso e allo stesso tempo vuole essere l'altro da sé, perciò è due volte assente da se stesso e in questo si potrebbe dire che è uomo per eccellenza. Che è due volte uomo"24. Di qui ha origine quella tensione tipicamente ebraica, che diventa "tensione creatrice e la sua soluzione risiede all'infinito" 25 . Il che vale a dire che tale tensione si risolve nel tempo e nel movimento. Il messianismo ebraico incarna perfettamente questa concezione del tempo come movimento infinito. 
Il rifiuto dell' idea della fine dei tempi e quindi della conclusività del nostro operare ancora una volta ci riporta alla musica di Debussy, come metafora dell'esistenza umana ed ebraica in particolare. La fine dei tempi è la morte così come l'indicibile è ancora metafora della morte, della paralizzante immobilità a cui conduce. Il Messia come tensione verso il futuro, come speranza indefinita e ineffabile è invece la vita, il movimento infinito, il tempo nella sua più profonda dinamica: ma questo discorso, va notato ancora una volta, è proprio della musica come dell'ebraismo! e non a caso Jankélévitch usa termini chiave come innocenza per designare il procedere dell'ebreo come del musicista. Si potrebbe dire che l'innocenza è il modo più appropriato di vivere il tempo come movimento ed è una qualità ugualmente necessaria al musicista come all'ebreo. Solo nell' innocenza del compositore così come dell'interprete, la musica può esercitare il suo potere e la sua suggestione profonda, la sua "força persuasiva", trascinando l'ascoltatore nel flusso del suo movimento. E' la stessa innocenza necessaria "all'ebreo che va avanti, che raggiunge in questo modo l'innocenza", lontano dalla tentazione impura, l'innocenza che "ignora la lotta", l'innocenza che "essendo nel movimento, implica essa stessa la serenità $\mathrm{e}$ l'indifferenza..." "26. Lo stato d'animo più consono al musicista ed anche all' ebreo è dunque lo scherzo e l'humour: è l'unica possibile serietà, l'unica possibile saggezza per l'uomo. Così come l'ebreo non può che ironizzare sottilmente sull'ambiguità della propria situazione esistenziale, così il musicista, che ritrova un'analoga ambiguità nell'essenza stessa della musica ricorre all'humour per esprimere il contenuto più profondo della musica stessa che, come afferma efficacemente Jankélévitch, è al tempo stesso "seria e frivola, profonda e superficiale"27.

Sono molti dunque i punti di contatto tra l'ebraismo, quello vissuto ed esperito da Jankélévitch, e la musica, la cui essenza più profonda s'incarna nell'opera di Debussy: si tratta di due esperienze limite che rimandano anzitutto alla situazione umana, una situazione da cui l'uomo tende a sfuggire per rifugiarsi in miti collettivi consolatori, vani e ingannevoli. In qualche modo l'ebraismo e la 
musica si pongono nel pensiero di Jankélévitch come due residui baluardi, silenziosi, appartati e soprattutto marginali alle "ebbrezze razionalistiche e dialettiche". Saper vivere la marginalità nella sua positività, come dimensione della vita appartata dai clamori e dalle troppo facili certezze può essere appunto il messaggio più pregnante che musica ed ebraismo, in quanto esperienze intensificate dell'esistenza umana, ci trasmettono, lontano dalle folle vocianti, come "reminiscenza o profezia", che può ricordare "all'uomo il mistero che egli porta in se stesso" 28

\section{NOTAS}

1. E. Lisciani-Petrini, L'apparenza e le forme. Filosofia e musica, in Jankélévitch, Nuove ed. Tempi Moderni, Napoli 1991, p. 110-111.

2. Idem, Introd. a Wladimir Jankélévitch, La musica e l'ineffabile, Tempi Moderni, 1985, p. XXXV.

3. Cfr. soprattuto la sua raccolta di scritti Sources, Editions du Seuil, Paris 1984; si veda inoltre la traduzione italiana parziale di questi scritti $L a$ coscienza ebraica, Editrice La Giuntina, Firenze 1986.

4. Cfr. La coscienza ebraica, Editrice La Giuntina, Firenze 1986, p. 28.

5. Ibidem, p. 23.

6. Ibidem, p. 28.

7. Ibidem, p. 36 .

8. Ibidem, p. 34-35.

9. Ibidem, p. 35.

10. Ibidem, p. 35-36.

11. Ibidem, p. 22.

12. Ibidem, p. 40.

13. Ibidem, p. 42-43.

14. Ibidem, p. 58.

15. Ibidem, p. 64-65.

16. Ibidem, p. 88-89.

17. Ibidem, p. 92.

18. Ibidem, p. 92-93.

19. Ibidem, 92-94.

20. Jankélévitch, La musica e l'ineffabile, p. 103-104.

21. Ibidem, p. 93.

22. Ibidem, p. 101.

23. La coscienza ebraica, p. 8.

24. Ibidem, p. 8. 
25. Ibidem, p. 22.

26. Tbidem, p. 93.

27. La musica e l'ineffabile, p. 91.

28. Ibidem, p. 211.

Enrico Fubini é ensaísta, escritor e Professor no Departamento de Música da Universidade de Turim. 